\title{
CC chemokine receptor 5 gene polymorphisms in beryllium disease
}

\author{
H. Sato*,\#, L. Silveira*, P. Spagnolo* , M. Gillespie*, E.B. Gottschall*, ${ }^{*}$, , K.I. Welsh", \\ R.M. du Bois ${ }^{\star, \#}$, L.S. Newman ${ }^{\uparrow,+}$ and L.A. Maier*, ${ }^{\star},+$
}

ABSTRACT: CC chemokine receptor 5 (CCR5) is expressed on type-1 T-helper cells, which are involved in the pathogenesis of the granulomatous lung disease chronic beryllium disease (CBD). CCR5 gene (CCR5) polymorphisms are associated with sarcoidosis severity. The present study explores associations between CCR5 polymorphisms and CBD and its disease progression.

Eight CCR5 polymorphisms were genotyped in CBD $(n=88)$, beryllium sensitisation (BeS; $n=86)$ and beryllium-exposed nondiseased controls $(n=173)$ using PCR with sequence-specific primers. Pulmonary function and bronchoalveolar lavage data were examined for associations with genotypes.

There were no significant differences in genotype and allele frequency between CBD, BeS individuals and controls. In CBD, associations were found with decline in forced expiratory volume in $1 \mathrm{~s}$ and forced vital capacity and the CCR5 -3458 thymidine (T)T genotype $(p<0.0001)$, and an increase in alveolar-arterial oxygen tension difference at rest $(p=0.003)$ and at maximum exercise $(p=0.01)$ and the -5663 adenine allele. Increased bronchoalveolar lavage lymphocyte numbers were associated with CCR5 -2459 guanine/-2135T $(p=0.01)$ only in the combined CBD and BeS group.

This is the first study showing that CCR5 polymorphisms are associated with worsening pulmonary function over time in CBD, suggesting that CCR5 is important in the progression of pulmonary function in CBD. Further studies would be useful to clarify the mechanism whereby CCR5 polymorphisms affect progression of CBD.

KEYWORDS: Broncholaveolar lavage, $\mathrm{CC}$ chemokine receptor 5, chronic beryllium disease, lung function tests, polymorphism

B eryllium exposure in the workplace triggers a beryllium-specific immune response in a minority of those exposed. Specifically, 2-10\% of exposed workers develop beryllium sensitisation (BeS), with lymphocytes that proliferate in response to beryllium in the beryllium lymphocyte proliferation test (LPT) (BeLPT) [1, 2]. Previous studies have shown that beryllium-stimulated T-cell proliferation is restricted to major histocompatibility complex class II [3]. Subsequently, trafficking of immune and inflammatory cells is initiated along with cytokine production, resulting in granuloma formation, the hallmark of chronic beryllium disease (CBD).

The chemokines involved in granuloma formation in CBD are unknown. The CC chemokine receptor 5 (CCR5) gene (CCR5), located on chromosome 3 p21 and adjacent to CCR2, is implicated in the chemotaxis and activation of leukocyte subsets, including CD4+ type-1 Thelper lymphocytes [4]. CCR5 is polymorphic and some of the variants appear to be functional, although this is not well understood $[5,6]$.

Of relevance to $\mathrm{CBD}$, an association between CCR5 polymorphisms and sarcoidosis was previously reported [7]. Although an association between CCR5 polymorphisms and presence of disease in sarcoidosis compared to controls was not found, an association between a specific CCR5 haplotype (human haplogroup C (HHC)) and chest radiographic stage of disease was found. Others have reported an increased frequency of a CCR5 32-bp deletion mutation $(\triangle 32)$ in sarcoidosis [8]. This may suggest that CCR5 polymorphisms are a risk factor for disease or disease severity in granulomatous lung diseases.
AFFILIATIONS

${ }^{*}$ Robert H. Hollis Laboratory of

Environmental and Occupational Health, Division of Environmental and Occupational Health Sciences, Dept of Medicine, National Jewish Health, Denver,

"Division of Pulmonary and Critical Care Sciences, Dept of Medicine, School of Medicine, University of

Colorado Denver, and

${ }^{+}$Dept of Environmental and Occupational Health, School of Public Health, University of Colorado Denver, Denver, C0, USA. ${ }^{\#}$ Clinical Genomic Group, Royal Brompton Hospital and National Heart and Lung Institute, Imperial College London, London, UK.

CORRESPONDENCE

L.A. Maier

National Jewish Medical Health 1400 Jackson Street

Denver

CO 80206

USA

E-mail: maier@@nhhealth.org

Received:

July 102009

Accepted after revision:

Dec 222009

First published online: Jan 142010 
An increase in CCR5 expression has been reported on bronchoalveolar lavage (BAL) cells in sarcoidosis [9-11]. There are no reports of an association between CCR5 polymorphisms and CBD, which is histopathologically identical to sarcoidosis, sharing many of the clinical and radiographic features of sarcoidosis. Against this background, it was hypothesised that CCR5 polymorphisms and/or haplotypes would be associated with CBD and more severe forms of disease, but not with BeS, in which pulmonary granulomatous disease is absent. The aims of the present study were to: 1) assess the contribution of CCR5 polymorphisms and/or haplotypes as risk factors for $\mathrm{CBD}$ compared to $\mathrm{BeS}$ and beryllium-exposed nondiseased controls; 2) evaluate the association between these polymorphisms and severity markers in $\mathrm{CBD}$; and 3) investigate the association between BAL cellularity and CCR5 polymorphisms and/or haplotypes, in order to determine whether or not there is a functional association between CCR5 polymorphisms and CBD.

\section{METHODS}

\section{Study design}

A case-control study was conducted that compared Caucasian subjects with CBD $(n=88)$ and $\operatorname{BeS}(n=86)$ and subjects with beryllium exposure without evidence of sensitisation or disease (controls; $\mathrm{n}=173$ ) in order to evaluate CCR5 polymorphisms and their association with CBD. Only Caucasian cases and controls were utilised in order to limit population stratification, as the number of non-Caucasians (e.g. black Americans, Asians, etc.) was too small to permit meaningful analysis. A case comparison study was used to evaluate CCR5 polymorphisms and disease severity in CBD. Cases were enrolled from the clinic at National Jewish Health (Denver, CO, USA). Cases of CBD and BeS had evidence of two abnormal blood BeLPTs. If the cases showed evidence of either noncaseating granulomas or a mononuclear cell infiltrate on lung biopsy, or BAL cell lymphocytosis of $\geqslant 15 \%$ and an abnormal BAL cell LPT on bronchoscopy, they were diagnosed as having CBD. If they showed no evidence of these abnormalities, they were diagnosed as having BeS. Controls were undergoing medical surveillance in the same beryllium industries as cases [12-14], and had undergone at least one normal BeLPT. Study subjects provided informed consent according to a protocol approved by the Institutional Review Board at National Jewish Health.

Cases of CBD and BeS underwent venepuncture, a BeLPT, preand post-bronchodilator pulmonary function testing and maximum exercise testing with an indwelling arterial line during initial (baseline) and subsequent clinical evaluations [15]. These data were obtained in the Pulmonary Physiology Unit at National Jewish Health using the same clinical protocols over time on the same equipment. Post-bronchodilator pulmonary function variables were used for analysis. Bronchoscopy with BAL, transbronchial biopsy and BAL LPTs were performed for clinical purposes [15]. Control subjects underwent venepuncture and BeLPTs. All subjects completed a modified version of the American Thoracic Society questionnaire [15].

\section{CCR5 genotypes}

Genomic DNA was extracted from whole blood using the PAXgene kit (Qiagen, Inc., Valencia, CA, USA). PCR with sequence-specific primers [16, 17] was used to identify eight CCR5 single nucleotide polymorphisms (SNPs): -5663 adenine (A)/guanine (G) (SNP identifier rs2040388), -3900 cytosine (C)/ A (rs2856757), -3458 thymidine (T)/G (rs2734225), -2459G/ A (rs1799987), -2135T/C (rs1799988), -2086A/G (rs1800023), $-1835 \mathrm{C} / \mathrm{T}$ (rs1800024), and $\Delta 32$, which had been investigated in a previous study on sarcoidosis [7].

\section{Data analysis}

All genotype frequencies were tested for Hardy-Weinberg equilibrium using Chi-squared goodness-of-fit tests. Normalising transformations were performed on continuous outcome variables when necessary to better approximate model assumptions; these data are expressed as geometric mean \pm geometric SD (GSD). Measures of linkage disequilibrium, the normalised disequilibrium constant $\left(D^{\prime}\right)$ and $r^{2}$, were calculated using Haploview (Whitehead Institute for Biomedical Research, Cambridge, MA, USA). Haplotype blocks were determined with Haploview using the confidence interval (CI) method [18]. Haplotype frequencies were estimated using Haplo.Score [19]. In order to adjust for uncertainty in haplotype assignments, weighted logistic regression models were used to assess associations with disease and disease severity. Comparisons were made when sufficient numbers of observed haplotypes were available. Linear regression was used to determine the influence of genotype/haplotype on cross-sectional continuous outcomes. Mixed-effects models were used to assess longitudinal outcomes and to estimate effects based on different numbers of follow-up visits and different timings of the visits. Age at testing, sex, smoking history, height and time from first beryllium exposure were included in the model, along with the genotype/haplotype and forced expiratory volume in $1 \mathrm{~s}$ (FEV1), forced vital capacity (FVC), total lung capacity (TLC), diffusing capacity of the lung for carbon monoxide uncorrected for haemoglobin (DL,CO,uncor), arterial oxygen tension at rest $\left(\mathrm{Pa}_{2} \mathrm{O}_{2}, \mathrm{r}\right)$ and during maximal exercise $\left(\mathrm{Pa}_{2} \mathrm{O}_{2}, \mathrm{max}\right)$, alveolar-arterial oxygen tension difference at rest $\left(P \mathrm{~A}-\mathrm{a}, \mathrm{O}_{2}, \mathrm{r}\right)$ and during maximal exercise $\left(P \mathrm{~A}-\mathrm{a}, \mathrm{O}_{2}, \mathrm{max}\right)$, and workload at maximal exercise ( $W$ max $)$. The variable time from first exposure to visit date was restricted to visits ranging 10-40 yrs from first exposure, since there were few visits outside this range from which to make estimates. In order to avoid effects of corticosteroids on the severity of CBD, all subjects' data were included until commencement of corticosteroid use. No adjustment was made for multiple comparisons since this adjustment is too conservative, with the high correlation of outcomes. Statistical analysis was performed using Knowledge STUDIO (Angoss Software Corporation, Toronto, Canada) or SAS software version 9.1 (SAS Institute, Inc., Cary, NC, USA). All tests were two sided and a $\mathrm{p}$-value of $<0.05$ was used to determine significance.

\section{RESULTS}

\section{Demographic information}

There were no significant sex differences between the three study groups. The control subjects $(60.5 \pm 11.0 \mathrm{yrs})$ were older than both the CBD $(54.1 \pm 10.8 \mathrm{yrs})$ and BeS subjects $(53.5 \pm 12.0 \mathrm{yrs}) \quad(\mathrm{p}<0.0001)$. Ever-smoking was not significantly different across the groups. In the CBD group, 18 $(20.5 \%)$ patients had received corticosteroids. The BeS subjects and controls were not treated with corticosteroids. 


\section{CCR5 polymorphisms}

CCR5 promoter polymorphisms were compared across groups, as summarised in table 1 . All populations were in HardyWeinberg equilibrium for all genotypes. CCR5 -2459A/G and $-2135 \mathrm{C} / \mathrm{T}$ were in $100 \%$ linkage disequilibrium $\left(D^{\prime}=1\right)$. CCR5 $-3900 \mathrm{~A}$ (odds ratio (OR) $0.35 ; 95 \%$ CI $0.14-0.84 ; \mathrm{p}=0.03$ ), $-3458 \mathrm{G}$ (OR 0.29; 95\% CI 0.11-0.78; $\mathrm{p}=0.02$ ) and $-2459 \mathrm{~A} /-2135 \mathrm{C}$ (OR 0.39; 95\% CI 0.18-0.86; $\mathrm{p}=0.03$ ) were decreased in CBD

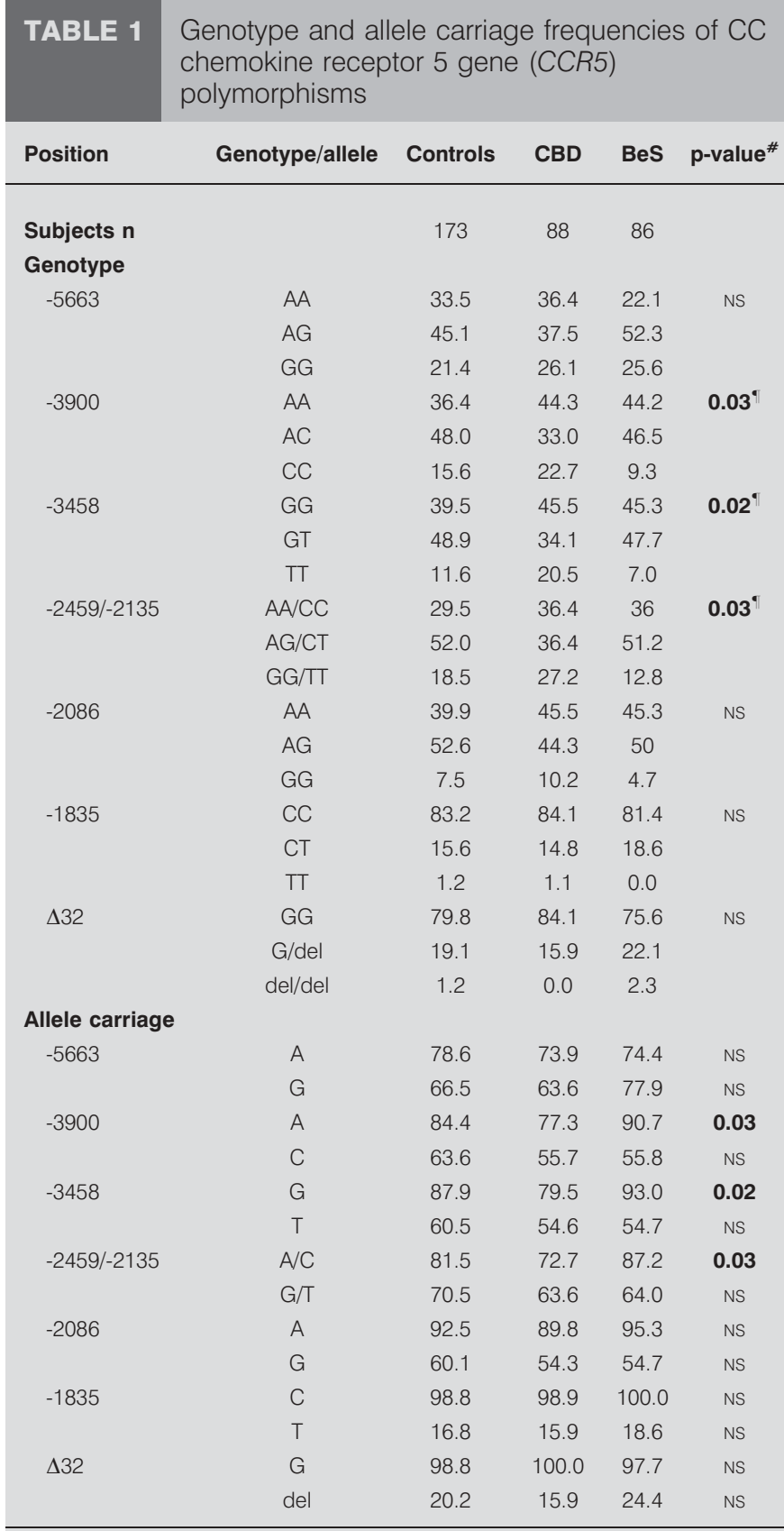

Data are presented as percentages. CBD: chronic beryllium disease; BeS: beryllium sensitisation; $\triangle 32$ : CCR5 32-bp deletion mutation; A: adenine; $G$ : guanine; C: cytosine; T: thymidine; del: deletion; NS: nonsignificant. ${ }^{\#}: \mathrm{CBD}$ versus BeS; " : obtained using $2 \times 3$ tables and comparing genotype numbers in $\mathrm{CBD}$ and $\mathrm{BeS}$. compared to BeS. However, no significant differences were found between CBD and controls, nor between BeS and controls. Consistent with the allele frequencies, similar differences were found between $\mathrm{CBD}$ and $\mathrm{BeS}$ when comparing the genotypes at the same three positions (table 1). No other associations were found between any of the other CCR5 polymorphisms and disease.

The haplotype comprising the -5663/-3900/-3458/-2459/-2135/ -2086 SNPs was identified using the CI method in Haploview (fig. 1). Table 2 shows the frequency of common (>1\%) CCR5 haplotypes. There were no significant differences in CCR5 haplotype between the three groups.

\section{Longitudinal analysis of disease severity in CBD}

Mixed-effects models were used to fit lung function measurements from clinical visits, including FEV1, FVC, TLC, $D \mathrm{~L}, \mathrm{CO}$, uncor, $\mathrm{Pa}_{2} \mathrm{O}_{2}, \mathrm{r}, \mathrm{Pa}_{2} \mathrm{O}_{2}, \max , P \mathrm{~A}-\mathrm{a}, \mathrm{O}_{2}, \mathrm{r}, P A-\mathrm{a}, \mathrm{O}_{2}$, $\max$ and $W_{\max }$ and covariates, as noted above, along with CCR5 variants. The mean number of visits was 3.96 , with a maximum of 15 . The first visit was, on average, 18.1 \pm 8.2 yrs from first exposure and the last visit, on average, $27.2 \pm 8.8$ yrs. Tables 3 and 4 show the mean rates of change in these variables over time in relation to the CCR5 genotypes of interest, adjusting for age at testing, sex, smoking history, height and time from first beryllium exposure.

On average, CBD subjects homozygous for -5663 A showed a significantly greater decrease in FEV1 over time $(p=0.01)$ than
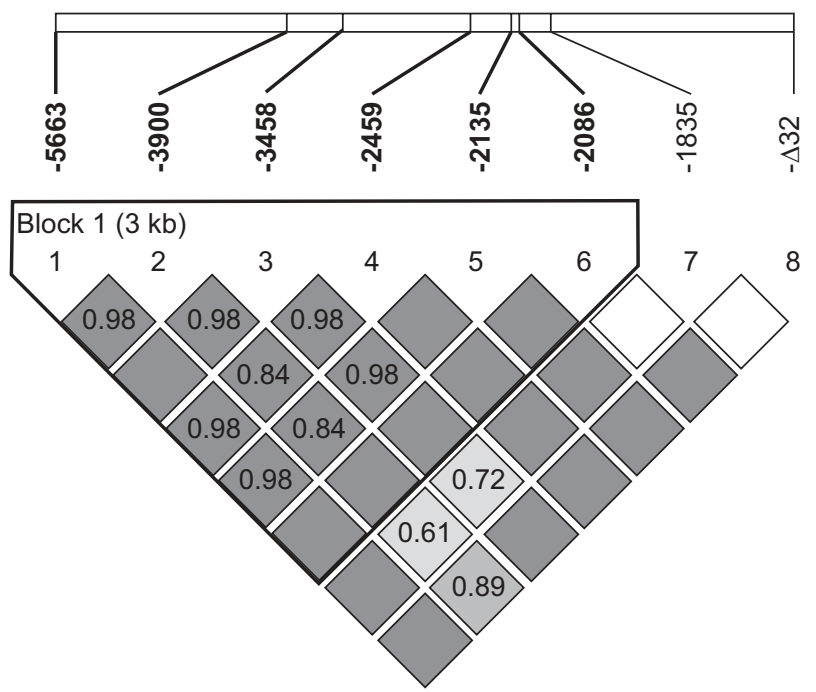

FIGURE 1. CC chemokine receptor 5 gene linkage disequilibrium and haplotype block structure in cases and controls derived using the confidence interval method. Two measures of pairwise linkage disequilibrium used in the Haploview program are normalised disequilibrium constant $\left(D^{\prime}\right)$ and logarithm of odds (LOD) scores. Pairwise linkage disequilibrium plots demonstrate the $D^{\prime}$ for the eight polymorphisms in Caucasian cases and controls. The numbers shown represent the $D^{\prime}$ for adjacent pairs; empty squares indicate a $D^{\prime}$ of 1.0 ( $\square$ : greatest linkage disequilibrium, with an upper confidence bound ( $\mathrm{UCl}$ ) of $D^{\prime}$ of 1.0 and an LOD score of $>2$; 1 : intermediate linkage disequilibrium, with a $U C l$ of $D^{\prime}$ of 1.0 and an LOD score of $\sim 2$; $:$ : less information about linkage disequilibrium, with a $\mathrm{UCl}$ of $D^{\prime}$ of 1.0 but an LOD score of $<2, \square$ : UCl of $<1.0$ for $D^{\prime}$ and an LOD score of $<2$ ). Using the confidence interval method, a haplotype block was identified between the $-5663,-3900,-3458,-2459,-2135$ and -2086 polymorphisms 


\begin{tabular}{|c|c|c|c|c|c|c|c|c|c|}
\hline TABLE 2 & $\begin{array}{l}\text { Common C } \\
\text { sensitisatior }\end{array}$ & $\begin{array}{l}\text { mokin } \\
\text { ) and }\end{array}$ & $\begin{array}{l}\text { ptor } 5 \\
\text { ls }\end{array}$ & haplo & equenc & chron & ium dise & D), & \\
\hline Haplotype & -5663 & -3900 & -3458 & -2459 & -2135 & -2086 & Controls & CBD & $\mathrm{BeS}$ \\
\hline Subjects $n$ & & & & & & & 346 & 164 & 172 \\
\hline 1 & G & A & G & A & C & A & 43.2 & 44.3 & 50.6 \\
\hline 2 & A & C & T & G & T & G & 34.0 & 32.4 & 29.0 \\
\hline 4 & A & A & G & G & T & A & 7.8 & 7.9 & 7.4 \\
\hline 5 & A & C & G & A & C & A & 3.1 & 1.1 & 1.1 \\
\hline 6 & A & C & $\mathrm{T}$ & $G$ & $\mathrm{~T}$ & A & 1.7 & 4.5 & 1.2 \\
\hline
\end{tabular}

Data are presented as percentages; only the common haplotypes (>1\%) are shown. A: adenine; G: guanine; C: cytosine; T: thymidine.

did nonhomozygous subjects (fig. 2a). Carriers of the A allele also showed a greater decline in FEV1 than noncarriers $(\mathrm{p}=0.001)$ (table 3). A similar trend was seen with these same alleles in relation to FVC. Carriers of the $-5663 \mathrm{~A}$ allele showed a greater decline in $\mathrm{Pa}_{\mathrm{a}} \mathrm{O}_{2}, \mathrm{r}(\mathrm{p}=0.01)$, as well as an increase in $\log P \mathrm{~A}-\mathrm{a}, \mathrm{O}_{2}, \mathrm{r}$ $(\mathrm{p}=0.003)$ and $\log P \mathrm{~A}-\mathrm{a}, \mathrm{O}_{2}, \max (\mathrm{p}=0.01)$, than did the noncarriers (table 4). Subjects homozygous for the $-3900 \mathrm{C}$ allele exhibited greater FEV1 $(p=0.0002)$ and FVC decline $(p<0.0001)$ and a

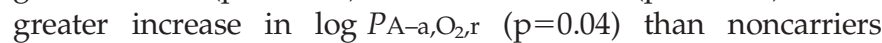
(tables 3 and 4). Subjects homozygous for $-3458 \mathrm{~T}$ showed greater FEV1 $(p<0.0001)$ and FVC declines $(p<0.0001)$ than nonhomozygous subjects. Those homozygous for $-2459 \mathrm{G} /-2135 \mathrm{~T}$ (the two loci in complete linkage disequilibrium) exhibited lower FEV1 $(p=0.04)$ and FVCs $(p=0.02)$ than nonhomozygous subjects. Subjects with at least one $\Delta 32$ allele showed lower FEV1 $(p=0.002)$ and FVCs $(p=0.008)$ than noncarriers.
The impact of CCR5 haplotype on change in lung function over time was evaluated and similar results were noted (tables 3 and 4). Carriers of haplotype 1 (-5663G/-3900A/-3458G/-2459A/$2135 \mathrm{C} /-2086 \mathrm{~A})$ showed a lesser decline in FEV1 $(\mathrm{p}<0.0001)$, FVC $(\mathrm{p}=0.0004)$ (fig. $2 \mathrm{~b})$, TLC $(\mathrm{p}=0.01)$ and $\mathrm{Pa}_{\mathrm{a}} \mathrm{O}_{2}, \mathrm{r}(\mathrm{p}=0.01)$ (fig. 2c) than did noncarriers. Carriers of haplotype 2 (-5663A/$3900 \mathrm{C} /-3458 \mathrm{~T} /-2459 \mathrm{G} /-2135 \mathrm{~T} /-2086 \mathrm{G})$ demonstrated increasing $\log P \mathrm{~A}-\mathrm{a}, \mathrm{O}_{2}, \mathrm{r}(\mathrm{p}=0.03)$ and $\log P \mathrm{~A}-\mathrm{a}, \mathrm{O}_{2}, \max (\mathrm{p}=0.01)$ and $\mathrm{a}$ greater decline in FEV1 $(p=0.005)$ over time than noncarriers.

\section{CCR5 polymorphisms and BAL lymphocytes}

Comparisons were made between BAL differential cell counts at the first and last visits to clinic of $88 \mathrm{CBD}$ and $78 \mathrm{BeS}$ subjects. There were significant differences between geometric mean \pm GSD lymphocyte percentages in BAL from CBD $(24.3 \pm 2.35 \%$; $n=88)$ compared with BeS $(9.3 \pm 2.24 \%$; $n=78$;

TABLE 3 Estimates of change in lung function indices by CC chemokine receptor 5 gene (CCR5) polymorphism and haplotype in chronic beryllium disease ${ }^{\#}$

\begin{tabular}{|c|c|c|c|c|c|c|c|c|}
\hline Carrier & FEV 1 L.yr $r^{-1}$ & $p$-value & FVC L.yr ${ }^{-1}$ & p-value & $\mathrm{TLC} L \cdot \mathrm{yr}^{-1}$ & $\mathrm{p}$-value & $\begin{array}{c}D \mathrm{~L}, \mathrm{CO}, \mathrm{uncor} \\
\mathrm{mL} \cdot \mathrm{min}^{-1} \cdot \mathrm{mmHg}^{-1} \cdot \mathrm{yr}^{-1}\end{array}$ & $p$-value \\
\hline$-5663 A$ & $-0.03 \pm 0.009)$ & 0.001 & $-0.02 \pm 0.01$ & 0.12 & $-0.006 \pm 0.01$ & 0.61 & $0.08 \pm 0.11$ & 0.48 \\
\hline$-3900 \mathrm{CC}$ & $-0.03 \pm 0.008$ & 0.0002 & $-0.04 \pm 0.01$ & $<0.0001$ & $-0.02 \pm 0.01$ & 0.22 & $-0.05 \pm 0.11$ & 0.64 \\
\hline$-3900 C$ & $-0.02 \pm 0.007$ & 0.007 & $-0.02 \pm 0.01$ & 0.06 & $-0.004 \pm 0.01$ & 0.7 & $-0.01 \pm 0.09$ & 0.93 \\
\hline$-2459 G G /-2135 T T$ & $-0.02 \pm 0.008$ & 0.04 & $-0.03 \pm 0.009$ & 0.02 & $-0.01 \pm 0.01$ & 0.36 & $-0.09 \pm 0.10$ & 0.36 \\
\hline$-2459 G /-2135 T$ & $-0.01 \pm 0.01$ & 0.06 & $-0.006 \pm 0.01$ & 0.56 & $-0.02 \pm 0.01$ & 0.07 & $0.03 \pm 0.10$ & 0.74 \\
\hline$-2086 A A$ & $0.01 \pm 0.01$ & 0.12 & $0.009 \pm 0.01$ & 0.28 & $0.008 \pm 0.01$ & 0.4 & $0.0001 \pm 0.09$ & 0.99 \\
\hline$-1835 \mathrm{CC}$ & $0.008 \pm 0.02$ & 0.53 & $0.007 \pm 0.02$ & 0.68 & $0.01 \pm 0.02$ & 0.44 & $-0.17 \pm 0.16$ & 0.29 \\
\hline$\Delta 32$ & $-0.03 \pm 0.008$ & 0.002 & $-0.03 \pm 0.12$ & 0.008 & $-0.02 \pm 0.01$ & 0.12 & $-0.06 \pm 0.12$ & 0.58 \\
\hline
\end{tabular}

Data are presented as mean $\pm \mathrm{SD}$, and represent the mean increased rate of decline comparing carriage versus non-carriage of genotypes/alleles/haplotypes. Haplotype 1 is -5663G/-3900A/-3458G/-2459A/-2135C/-2086A; haplotype 2 is -5663A/-3900C/-3458T/-2459G/-2135T/-2086G. FEV1: forced expiratory volume in 1 s; FVC: forced vital capacity; TLC: total lung capacity; $D \mathrm{~L}, \mathrm{CO}$,uncor: diffusing capacity of the lung for carbon monoxide uncorrected for haemoglobin; $\mathrm{A}$ : adenine; C: cytosine; T: thymidine; G: guanine; $\triangle 32$ : CCR5 32-bp deletion mutation. ${ }^{\#:}$ 10-40 yrs from first beryllium exposure. 


\begin{tabular}{|c|c|c|c|c|c|c|c|c|c|c|}
\hline $\begin{array}{l}\text { TABLE } 4 \text { Es } \\
\text { ha }\end{array}$ & $\begin{array}{l}\text { ates of char } \\
\text { type in chro }\end{array}$ & $\begin{array}{l}\text { ge in fur } \\
\text { hic beryll }\end{array}$ & $\begin{array}{l}\text { her lung fun } \\
\text { im disease }\end{array}$ & tion ind & by CC $\mathrm{c}$ & nokine & eptor $5 \mathrm{c}$ & e $(C O$ & polymorp & and \\
\hline Carrier & $\begin{array}{c}\mathrm{Pa} \mathrm{O}_{2}, \mathrm{max} \\
\mathrm{mmHg} \cdot \mathrm{yr}^{-1}\end{array}$ & p-value & $\begin{array}{c}\mathrm{Pa}, \mathrm{O}_{2}, \mathrm{r} \\
\mathrm{mmHg} \cdot \mathrm{yr}^{-1}\end{array}$ & $p$-value & $\begin{array}{l}\mathrm{PA}-\mathrm{a}, \mathrm{O}_{2}, \mathrm{max} \\
\mathrm{mmHg} \cdot \mathrm{yr}^{-1}\end{array}$ & $\mathrm{p}$-value & $\begin{array}{c}P A-\mathrm{a}, \mathrm{O}_{2}, \mathrm{r} \\
\mathrm{mmHg} \cdot \mathrm{yr}^{-1}\end{array}$ & p-value & $W \max W \cdot y r^{-1}$ & p-value \\
\hline$-5663 A A$ & $-0.12 \pm 0.24$ & 0.62 & $-0.21 \pm 0.21$ & 0.33 & $1.01 \pm 1.02$ & 0.49 & $1.01 \pm 1.02$ & 0.49 & $0.36 \pm 0.71$ & 0.61 \\
\hline$-5663 A$ & $-0.13 \pm 0.20$ & 0.50 & $-0.40 \pm 0.16$ & 0.01 & $1.04 \pm 1.01$ & 0.01 & $1.06 \pm 1.02$ & 0.003 & $-0.73 \pm 0.56$ & 0.19 \\
\hline$-3900 \mathrm{C}$ & $0.15 \pm 0.20$ & 0.45 & $-0.08 \pm 0.17$ & 0.65 & $1.02 \pm 1.01$ & 0.07 & $1.04 \pm 1.02$ & 0.04 & $-0.13 \pm 0.57$ & 0.82 \\
\hline -3458TT & $0.11 \pm 0.23$ & 0.62 & $-0.23 \pm 0.17$ & 0.18 & $0.99 \pm 1.02$ & 0.45 & $1.00 \pm 1.02$ & 0.98 & $-1.8 \pm 0.65$ & 0.01 \\
\hline$-3458 \mathrm{~T}$ & $-0.07 \pm 0.19$ & 0.71 & $-0.06 \pm 0.17$ & 0.71 & $1.22 \pm 1.17$ & 0.21 & $1.17 \pm 1.12$ & 0.14 & $-0.31 \pm 0.56$ & 0.58 \\
\hline$-2459 G G /-2135 T T$ & $-0.005 \pm 0.21$ & 0.98 & $0.25 \pm 0.16$ & 0.11 & $1.00 \pm 1.02$ & 0.98 & $1.01 \pm 1.02$ & 0.79 & $-1.2 \pm 0.58$ & 0.05 \\
\hline$-2459 G /-2135 T$ & $-0.28 \pm 0.21$ & 0.17 & $-0.19 \pm 0.18$ & 0.30 & $1.02 \pm 1.01$ & 0.05 & $1.03 \pm 1.02$ & 0.06 & $0.14 \pm 0.60$ & 0.81 \\
\hline$-2086 A A$ & $0.07 \pm 0.19$ & 0.71 & $0.06 \pm 0.17$ & 0.70 & $1.02 \pm 1.02$ & 0.15 & $1.03 \pm 1.02$ & 0.09 & $0.31 \pm 0.56$ & 0.58 \\
\hline
\end{tabular}

$\mathrm{p}<0.0001)$ at the first visit to clinic, and for CBD $(28.0 \pm 1.87 \%$; $\mathrm{n}=51)$ compared with $\operatorname{BeS}(7.0 \pm 0.22 \% ; \mathrm{n}=47 ; \mathrm{p}<0.0001)$ at the last visit.

The relationship between BAL lymphocyte percentage and CCR5 polymorphisms was evaluated, comparing the CCR5 polymorphisms, haplotypes and BAL lymphocyte percentages in $\mathrm{CBD}$ and $\mathrm{BeS}$ combined at the first visit to clinic (table 5). The percentage of BAL lymphocytes was significantly increased in individuals carrying the CCR5 polymorphisms -2459G/-2135T $(17.99 \pm 2.53 \% ; n=104)$ compared with noncarriers $(12.30 \pm$ $2.64 \% ; n=62 ; p=0.01)$. Other CCR5 polymorphism associations were found with both increased and decreased numbers of lymphocytes for $\mathrm{CBD}$ and BeS subjects combined. When subjects with CBD and BeS were analysed separately, far fewer associations were demonstrated, although similar trends were apparent (table 1 of online supplementary material).

There was a significant difference in mean BAL lymphocyte percentage between carriers and noncarriers of haplotype 1 in the combined CBD and BeS group $(p=0.009)$ (table 5), with noncarriers of this haplotype showing higher lymphocyte percentages. This haplotype includes all alleles that were associated with a lower BAL lymphocyte percentage (-5663G/3900A/-3458G/-2459A/-2135C). Carriers of haplotype 2, which includes -2459A/-2135C, showed, on average, higher lymphocyte percentages than noncarriers of this haplotype in the combined CBD and BeS group. Similar, although nonsignificant, trends were seen in the BeS and CBD groups when analysed separately (table 1 of online supplementary material).

\section{DISCUSSION}

In the present study, the association between CCR5 polymorphisms in Caucasians with CBD and markers of disease severity is described. There were few significant differences in genotype, allele and haplotype frequency between either CBD or BeS and controls. Interestingly, a greater decline in lung function and worsening gas exchange was found in CBD cases carrying specific CCR5 polymorphisms and haplotypes. Associations were also shown between BAL lymphocyte percentages and CCR5 polymorphisms and haplotypes. Strikingly, these data support the fact that the same CCR5 polymorphisms associated with greater decline in CBD lung function are also associated with greater lymphocyte percentage in the lung. This suggests that CCR5 variants are functional, impacting lymphocyte recruitment into the CBD lung and, in turn, greater decline in lung function and worsening gas exchange.

This is the first study of CCR5 genotypes and haplotypes in CBD. Although the CCR5 -3900A, -3458G and -2459A/-2135C alleles were slightly decreased in $\mathrm{CBD}$ compared to BeS, no CCR5 polymorphisms or haplotype was significantly different between CBD and controls or BeS and controls. These findings suggest that CCR5 polymorphisms are not a risk factor for CBD. This is consistent with studies of sarcoidosis, a similar granulomatous lung disease of unknown aetiology. Although one study of Czech sarcoidosis cases found an increased rate of the $\Delta 32$ variant in cases compared to controls [8], two studies in UK/Dutch and German populations found no association with any CCR5 genotype or haplotype, including $\Delta 32$, and disease risk $[7,20]$. It should be noted that the frequencies of case and control genotypes reported in the present study are similar to those noted in these prior studies [7, 20].

In the current study, strong associations were found between changes in static lung function and measures of gas exchange and CCR5 polymorphisms in CBD. Specifically, the -5663AA, 

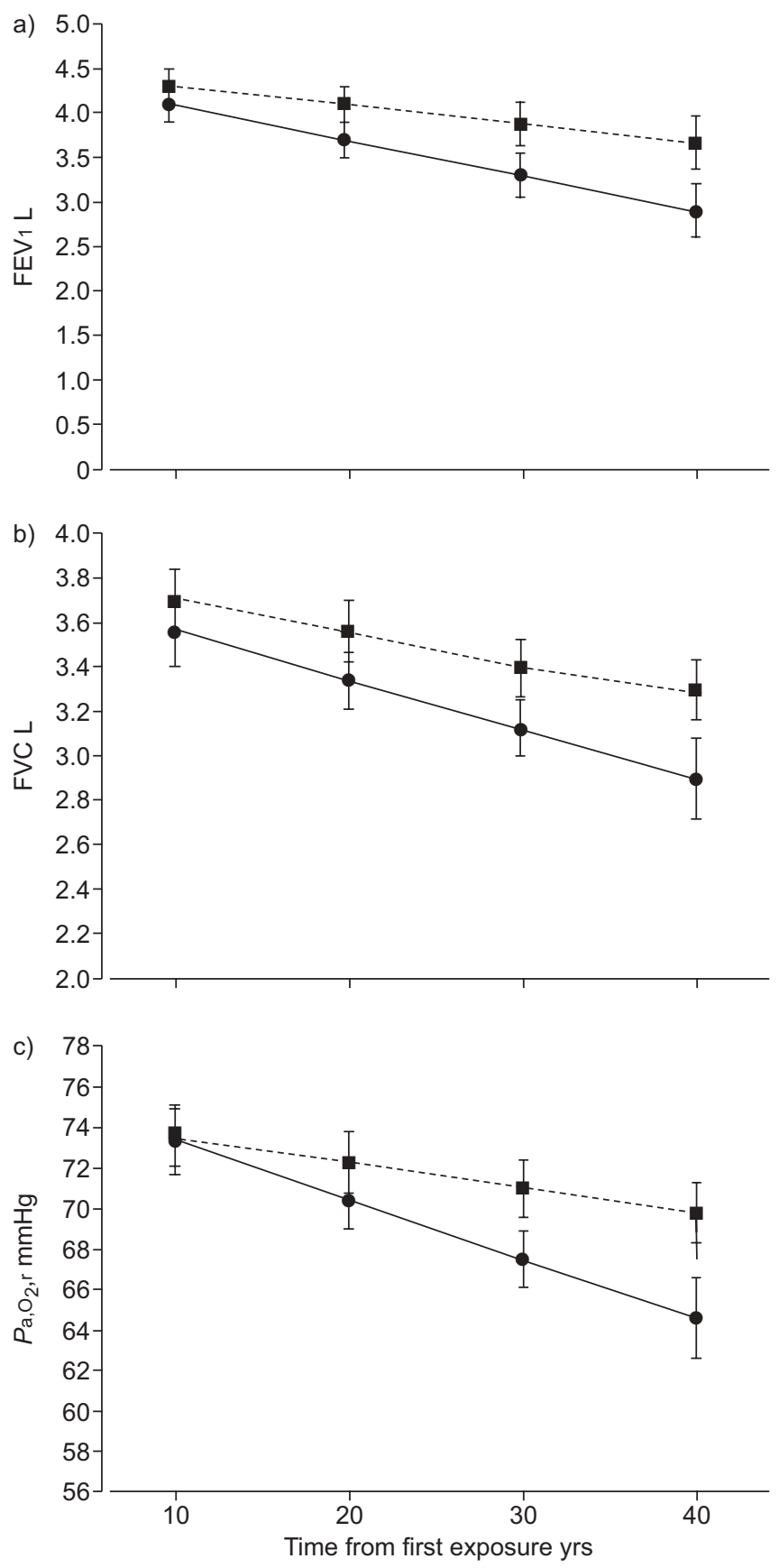

FIGURE 2. Mean change over time in: a) forced expiratory volume in $1 \mathrm{~s}$ (FEV1) in -5663 AA homozygotes (-) versus individuals with the -5663AG/GG genotype (------); and b) forced vital capacity (FVC); and c) arterial oxygen tension at rest $\left(\mathrm{Pa}_{\mathrm{a}} \mathrm{O}_{2}, \mathrm{r}\right)$ in those with at least one (-----) versus no (-) haplotype 1 (-5663G/-3900A/-3458G/-2459A/-2135C/-2086A), modelled ranging 10-40 yrs since first beryllium exposure and adjusted for sex, height ( $\mathrm{a}$ and $\mathrm{b}$ only), smoking history (current, former or never) and age at testing. Data are presented as mean \pm 2 SEM, and estimates are based on mixed-effects models. Individuals with the -5663AA genotype showed a greater decline in FEV1 than did the other genotypes $(p=0.01)$. There was a lesser decline in both $F V C$ and $P_{a}, O_{2}, r$ in haplotype 1 heterozygotes and homozygotes than in those with no haplotype 1 ( $p=0.01$ for both parameters).
-3900CC, -3458TT and -2459GG/-2135TT homozygous genotypes and carriage of the $\Delta 32$ allele were associated with a greater decline in FEV1 and FVC, whereas the -5663A and $-3900 \mathrm{C}$ alleles were associated with worsening measures of gas exchange at rest and during exercise. No associations were noted between any genotype and $\mathrm{DL}, \mathrm{CO}$, uncor, which may be due to the variability in the measurement of this parameter. Interestingly, some of the genotypes associated with worse lung function over time were the same as those noted to be slightly increased in CBD compared to BeS (-3900CC, -3458TT and -2459GG/-2135TT); this overrepresentation of genotypes in CBD may be due to the association with a more severe disease phenotype in the present CBD population rather than disease susceptibility. Most of the genotypes associated with greater decline in lung function were included in haplotype 2 (-5663A/-3900C/-3458T/-2459G/-2135T/-2086G). Carriers of this haplotype showed a greater decline in FEV1 and increase in $\mathrm{Pa}, \mathrm{O}_{2}, \mathrm{r}$ and $\mathrm{Pa}_{2} \mathrm{O}_{2}$, max.

The polymorphisms composing haplotype 2 in the current study are also represented in the HHC haplotype that was found to be overrepresented in higher sarcoidosis chest radiograph stages (stage II, III and IV) compared to lower stages (stage 0 and I) [7]. Similarly, the sarcoidosis HHC haplotype carriers also showed significantly lower FEV1 and FVCs on presentation compared with non-HHC carriers [7]. Conversely, the present CBD cases carrying -5663G/-3900A/ -3458G/-2459A/-2135C/-2086A (haplotype 1) exhibited better lung function, with higher FEV1, FVC, TLC and $\mathrm{Pa}_{2} \mathrm{O}_{2}, \mathrm{r}$ than noncarriers. These results suggest that CCR5 variants may be important in granulomatous lung disease progression, as indicated by chest radiography in sarcoidosis, and lung function changes in both sarcoidosis and CBD. Although a second CCR5 sarcoidosis study by FISCHER et al. [20] showed the CCR5 -5663A and -3900C alleles were increased in female patients with Löfgren's syndrome, they did not assess lung function or radiographic stage. As a result, it is difficult to compare our studies with theirs.

In addition to associations between lung function and CCR5 genotypes/haplotypes, associations were noted between BAL lymphocyte percentages and these same CCR5 genotypes/ haplotypes. Specifically, the BAL lymphocyte percentage was significantly increased in carriers of CCR5 -2459G/-2135T and haplotype 2 compared to noncarriers of these alleles. Conversely, carriers of CCR5 -5663G, -3900A, -3458G and $-2459 \mathrm{~A} /-2135 \mathrm{C}$ alleles and haplotype 1, containing all of these alleles, showed lower BAL lymphocyte percentages than noncarriers. It is possible that these data reflect functional alterations in CCR5 expression important in cell trafficking and cell-to-cell interactions. In this regard, CCR5 is expressed at high levels on CD4+ type-1 T-helper cells and may play a role in enhanced recruitment and activation of lymphocytes and monocytes to the lung and these cells' subsequent immune response [21]. It is possible that enhanced recruitment and activation of BAL lymphocytes may explain the association of the same CCR5 genotypes and haplotypes with more rapid disease progression.

Previously, KATCHAR et al. [10] reported a positive correlation between BAL lymphocyte percentages and the number of CCR5-expressing CD4+ BAL T-cells in sarcoidosis. CAPELLI 


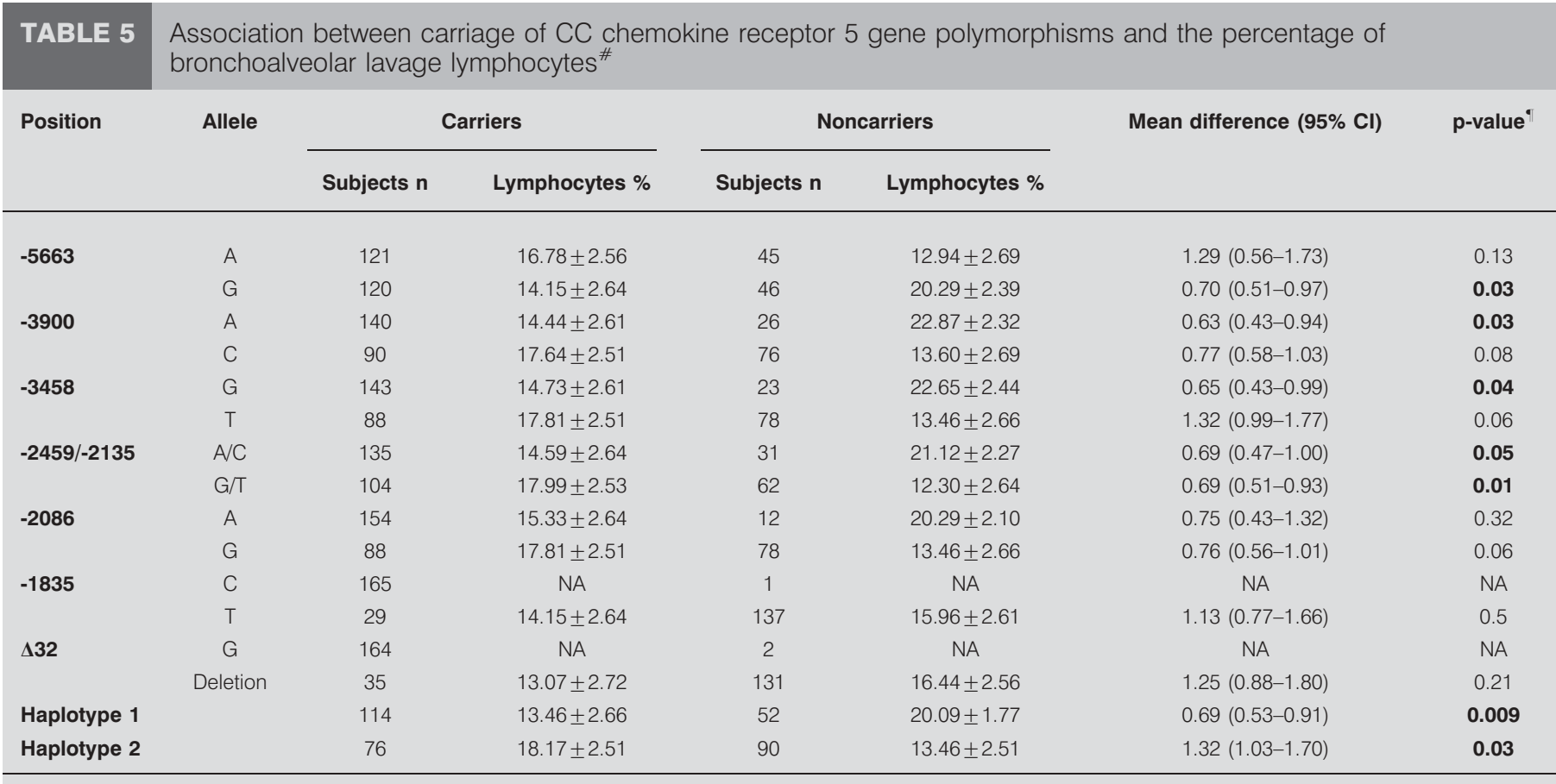

Data are presented as geometric mean \pm geometric sD unless otherwise indicated. Haplotype 1 is $-5663 \mathrm{G} /-3900 \mathrm{~A} /-3458 \mathrm{G} /-2459 \mathrm{~A} /-2135 \mathrm{C} /-2086 \mathrm{~A}$; haplotype 2 is -5663A/3900C/-3458T/-2459G/-2135T/-2086G. Cl: confidence interval; A: adenine; G: guanine; C: cytosine; T: thymidine; $\triangle 32$ : CCR5 32-bp deletion mutation; NA: not available \#: in chronic beryllium disease and beryllium sensitisation combined; " : unpaired t-test.

et al. [22] reported that increased CCR5 expression was observed in both lymphocytes and macrophages from all sarcoidosis patients, along with a trend towards decreased positivity from stage I to III of disease. They also showed that CCR5 expression was significantly reduced in lymphocytes from idiopathic pulmonary fibrosis compared to controls [9]. However, there have been no studies to date evaluating the association between CCR5 expression on BAL cells and CCR5 genotypes or haplotypes in sarcoidosis or other lung disease, including CBD.

The functional correlates of most of the CCR5 polymorphisms are unknown, except for the $\Delta 32$ deletion. Homozygosity for this 32-bp deletion results in truncation of the protein, meaning it lacks the transmembrane domain and thus cannot be expressed on the cell surface [23]. The CCR5 promoter polymorphism -2459G/A has been investigated primarily in HIV infection [6, 24-26]. Interestingly, the CCR5 -2459AA genotype has been associated with overexpression of CCR5 and HIV progression [6, 25]. This is discordant with the present findings. In order to clarify the discrepancy of associations with this CCR5 $-2459 \mathrm{G} / \mathrm{A}$ polymorphism between CBD and HIV infection, functional assay of CCR5 expression in CBD patients is needed, but such experiments are beyond the scope of the current study.

Although use of Bonferroni adjustment of the p-values for multiple comparisons made in the longitudinal analyses was considered, we chose not to do this as it would have been too conservative. That is to say, with the consistency of the correlations in the outcome variables, and the correlation between these variables, the Bonferroni adjustment would have overadjusted the present p-values. If only one or two significant associations had been found, these associations could have been discounted as possibly being due to chance alone because of the multiple comparisons undertaken in the present analysis. However, there were many associations found between disease severity indicators and CCR5 polymorphisms that all showed consistent direction, which supports our not using Bonferroni correction.

In summary, this is the first study to report associations between CCR5 polymorphisms and CBD. Although no overall association between CCR5 polymorphisms and either CBD or BeS was found, associations between worsening change in lung function and CCR5 polymorphisms and CCR5 haplotype 2 were found in CBD. Furthermore, the same variants were shown to be associated with increased BAL lymphocyte percentage, supporting the concept of a functional consequence of this genotype in CBD. Further studies are needed in order to show how these alterations in BAL effected worsening functional changes.

\section{SUPPORT STATEMENT}

This study was supported by P01 ES11810 from the National Institutes of Health (NIH; Bethesda, MD, USA), in part by University of Colorado Clinical and Translational Science Award grant 1 UL1 RR025780 from the NIH and National Center for Research Resources (Bethesda, MD, USA), and by the Asmarley Trust (London, UK).

\section{STATEMENT OF INTEREST}

None declared.

\section{ACKNOWLEDGEMENTS}

We would like to acknowledge the following for their contributions to the present work: G. Mondello for technical assistance; P. Mroz for 
helpful discussion; and C. Rodriguez for administrative support (all National Jewish Health, Denver, CO, USA); and, most importantly, the workers and patients who participate in these studies and make beryllium-related research possible.

\section{REFERENCES}

1 Mroz MM, Kreiss K, Lezotte DC, et al. Reexamination of the blood lymphocyte transformation test in the diagnosis of chronic beryllium disease. J Allergy Clin Immunol 1991; 88: 54-60.

2 Newman LS. Significance of the blood beryllium lymphocyte proliferation test. Environ Health Perspect 1996; 104: Suppl. 5, 953-956.

3 Saltini C, Winestock K, Kirby M, et al. Maintenance of alveolitis in patients with chronic beryllium disease by beryllium-specific helper T cells. N Engl J Med 1989; 320: 1103-1109.

4 Loetscher P, Uguccioni M, Bordoli L, et al. CCR5 is characteristic of Th1 lymphocytes. Nature 1998; 391: 344-345.

5 Kawamura T, Gulden FO, Sugaya M, et al. R5 HIV productively infects Langerhans cells, and infection levels are regulated by compound CCR5 polymorphisms. Proc Natl Acad Sci USA 2003; 100: 8401-8406.

6 McDermott DH, Zimmerman PA, Guignard F, et al. CCR5 promoter polymorphism and HIV-1 disease progression. Lancet 1998; 352: 866-870.

7 Spagnolo P, Renzoni EA, Wells AU, et al. C-C chemokine receptor 5 gene variants in relation to lung disease in sarcoidosis. Am J Respir Crit Care Med 2005; 172: 721-728.

8 Petrek M, Drabek J, Kolek V, et al. CC chemokine receptor gene polymorphisms in Czech patients with pulmonary sarcoidosis. Am J Respir Crit Care Med 2000; 162: 1000-1003.

9 Capelli A, Di Stefano A, Gnemmi I, et al. CCR5 expression and CC chemokine levels in idiopathic pulmonary fibrosis. Eur Respir J 2005; 25: 701-707.

10 Katchar K, Eklund A, Grunewald J. Expression of Th1 markers by lung accumulated $\mathrm{T}$ cells in pulmonary sarcoidosis. J Intern Med 2003; 254: 564-571.

11 Petrek M, Gibejova A, Drabek J, et al. CC chemokine receptor 5 (CCR5) mRNA expression in pulmonary sarcoidosis. Immunol Lett 2002; 80: 189-193.

12 Kelleher PC, Martyny JW, Mroz MM, et al. Beryllium particulate exposure and disease relations in a beryllium machining plant. J Occup Environ Med 2001; 43: 238-249.

13 Martyny JW, Hoover MD, Mroz MM, et al. Aerosols generated during beryllium machining. J Occup Environ Med 2000; 42: 8-18.
14 Newman LS, Mroz MM, Maier LA, et al. Efficacy of serial medical surveillance for chronic beryllium disease in a beryllium machining plant. J Occup Environ Med 2001; 43: 231-237.

15 Maier LA, Raynolds MV, Young DA, et al. Angiotensin-1 converting enzyme polymorphisms in chronic beryllium disease. Am J Respir Crit Care Med 1999; 159: 1342-1350.

16 Bunce M, O'Neill CM, Barnardo MC, et al. Phototyping: comprehensive DNA typing for HLA-A, B, C, DRB1, DRB3, DRB4, DRB5 \& DQB1 by PCR with 144 primer mixes utilizing sequence-specific primers (PCR-SSP). Tissue Antigens 1995; 46 355-367.

17 Welsh K, Bunce M. Molecular typing for the MHC with PCR-SSP. Rev Immunogenet 1999; 1: 157-176.

18 Gabriel SB, Schaffner SF, Nguyen $\mathrm{H}$, et al. The structure of haplotype blocks in the human genome. Science 2002; 296: 2225-2229.

19 Schaid DJ, Rowland CM, Tines DE, et al. Score tests for association between traits and haplotypes when linkage phase is ambiguous. Am J Hum Genet 2002; 70: 425-434.

20 Fischer A, Valentonyte R, Nebel A, et al. Female-specific association of $\mathrm{C}-\mathrm{C}$ chemokine receptor 5 gene polymorphisms with Löfgren's syndrome. J Mol Med 2008; 86: 553-561.

21 Grayson MH, Ramos MS, Rohlfing MM, et al. Controls for lung dendritic cell maturation and migration during respiratory viral infection. J Immunol 2007; 179: 1438-1448.

22 Capelli A, Di Stefano A, Lusuardi M, et al. Increased macrophage inflammatory protein- $1 \alpha$ and macrophage inflammatory protein$1 \beta$ levels in bronchoalveolar lavage fluid of patients affected by different stages of pulmonary sarcoidosis. Am J Respir Crit Care Med 2002; 165: 236-241.

23 Samson M, Labbe O, Mollereau C, et al. Molecular cloning and functional expression of a new human CC-chemokine receptor gene. Biochemistry 1996; 35: 3362-3367.

24 Hladik F, Liu H, Speelmon E, et al. Combined effect of CCR5- $\Delta 32$ heterozygosity and the CCR5 promoter polymorphism -2459 A/G on CCR5 expression and resistance to human immunodeficiency virus type 1 transmission. J Virol 2005; 79: 11677-84.

25 Knudsen TB, Kristiansen TB, Katzenstein TL, et al. Adverse effect of the CCR5 promoter -2459A allele on HIV-1 disease progression. J Med Virol 2001; 65: 441-444.

26 Salkowitz JR, Bruse SE, Meyerson H, et al. CCR5 promoter polymorphism determines macrophage CCR5 density and magnitude of HIV-1 propagation in vitro. Clin Immunol 2003; 108: 234-240. 